\title{
Short cuts
}

\author{
What's new in the other general journals
}

\section{Vitamins do not stop pre-eclampsia}

Academics from the United States have urged researchers to get back to basics in their search for a preventive treatment for pre-eclampsia, arguing in an editorial that limited research funds should be diverted away from expensive clinical trials until we know more about just what causes pre-eclampsia and how.

Large trials of various treatments have so far failed to deliver-including trials of aspirin, calcium supplements, and the antioxidant vitamins $\mathrm{C}$ and $\mathrm{E}$.

In the latest trial, antioxidant vitamins did not prevent pre-eclampsia in women at high risk and may even have made it worse. Pre-eclampsia happened earlier in women who took the vitamins, and their babies weighed less at birth than women who took a placebo. Worse, the supplements were associated with significantly more unexplained fetal deaths after 24 weeks (19/1393 (1\%) v 7/1391 (0.5\%); risk ratio $2.7,95 \%$ CI 1.0 to 7.1$)$.

Without a better understanding of the complex pathophysiology of pre-eclampsia, trials of preventive treatments are little more

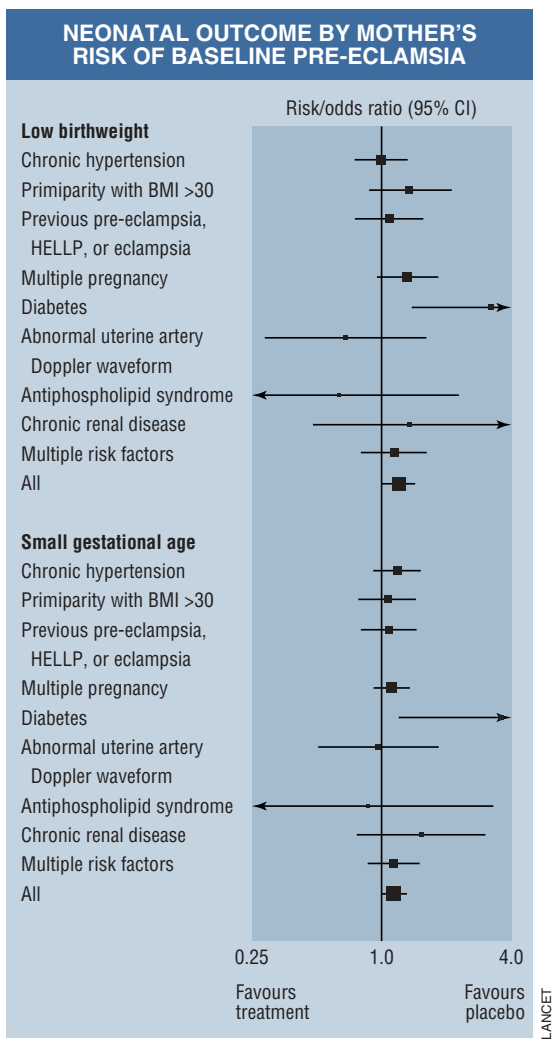

than a stab in the dark, says the editorial, even if they are driven by plausible theories and hopeful hints from small preliminary studies. But trials of antioxidant vitamins are already well underway. Their investigators have been warned.

Lancet 2006 Mar 30 (www.thelancet.com) doi 10.1016/S0140-6736(06)68434-1

\section{Being a mosaic protects women}

Men are generally more vulnerable to disease and death than women. Sex chromosomes are behind at least some of the difference, says one geneticist, who argues that women are protected from many diseases by their genetic mosaicism. Women have two X chromosomes, one from each parent. Men have only one, and it always comes from their mother. Although each of a woman's cells has only one working $\mathrm{X}$ chromosome (because one is disabled), can be from either parent, creating cellular diversity that protects women from disease. Ultimately, when women inherit a defective X linked gene, they have a back-up in reserve, even if it's expressed in only some of their cells.

To illustrate the point that it's bad for you to be monosomic for your mother's $\mathrm{X}$ chromosome, researchers studied serum lipid concentrations and the distribution of body fat in women with Turner's syndrome (pp 1373-4). Women with their mother's X chromosome, the equivalent of normal men, had more atherogenic lipid profiles, $36 \%$ (78 $v 58 \mathrm{ml}$ ) more abdominal fat, and 78\% (25v $14 \mathrm{ml}$ ) more visceral fat than women with their father's X chromosome. They effectively had male risk patterns for coronary heart disease.

JAMA 2006;295:1428-33

\section{HIV prevalence is falling in young people in southern India}

The best way to track the HIV pandemic is to look for changes in the incidence of infection. Because incidence of HIV is so hard to determine, researchers from India used prevalence in young people as a proxy measure. They found that between 2000 and 2004, the prevalence of HIV in women under 24 attending antenatal clinics fell from $1.7 \%$ to $1.1 \%$ in the south, a relative reduction of $35 \%$, or about $11 \%$ a year when adjusted for age $(\mathrm{P}<0.0001)$. The corresponding prevalence among young men aged 20 to 29 attending sexually

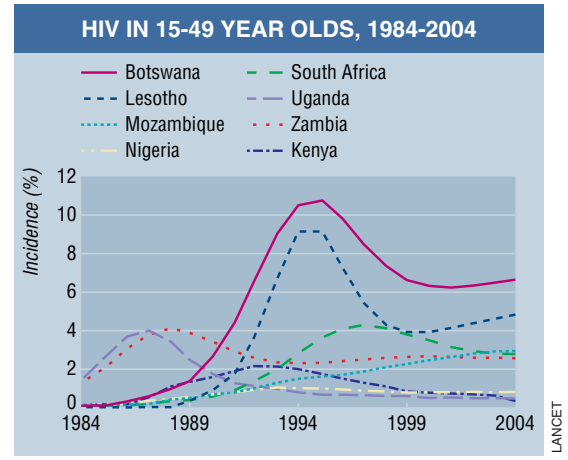

transmitted disease clinics also fell (from $21 \%$ to $13 \%$ ), again mostly in the south, where the epidemic is concentrated.

Although it's hard to say with certainty why these two groups of people have falling rates of HIV, the authors think that greater use of condoms by female sex workers and their clients is probably responsible. By 2004 , nearly $80 \%$ of female sex workers surveyed in two southern states said they used a condom with their last client.

Other parts of the world have good news too, says a linked editorial. The incidence of HIV is falling across Africa and has probably stabilised in China. Millions of people remain infected and many more will be infected, but the worldwide epidemic may at last have peaked.

Lancet 2006 Mar 30 (www.thelancet.com) doi: 10.1016/S0140-6736(06)68435-3

\section{Standard tests for genetic breast cancer are inadequate}

Screening for mutations in the breast cancer genes BRCA1 and BRCA2 helps women with a strong family history of breast or ovarian cancer to estimate their individual risk and decide how to manage it. A negative result is reassuring but doesn't completely rule out the chance of cancer causing mutations, a recent study has found.

Researchers studied 300 US citizens from high risk families. All had breast or ovarian cancer and had been cleared by standard commercial screening tests for BRCA1 and BRCA2 mutations. But a closer look using a technique called multiplex ligation dependent probe amplification found that the standard tests had missed mutations in $52(17 \%)$ of the women. Thirty one had rearrangements of BRCA1, four had rearrangements of BRCA2, and a further 17 had rearrangements in two other 
cancer causing genes-CHECK2 and TP53. Altogether the researchers found 28 different mutations. All were rare, and 14 were previously unknown.

Current testing options for women in families at high risk are inadequate, say the authors. The only commercially available test for BRCA1 and BRCA2 mutations detects only a few of the thousands of rearrangements that predispose women to cancer of the ovary and breast. Multiplex ligation dependent probe amplification is not available outside the confines of clinical trials. The authors say it should be.

\section{JAMA 2006;295:1379-88}

\section{New bird flu vaccine tested in humans}

\section{TESTING A POTENTIAL BIRD FLU VACCINE}

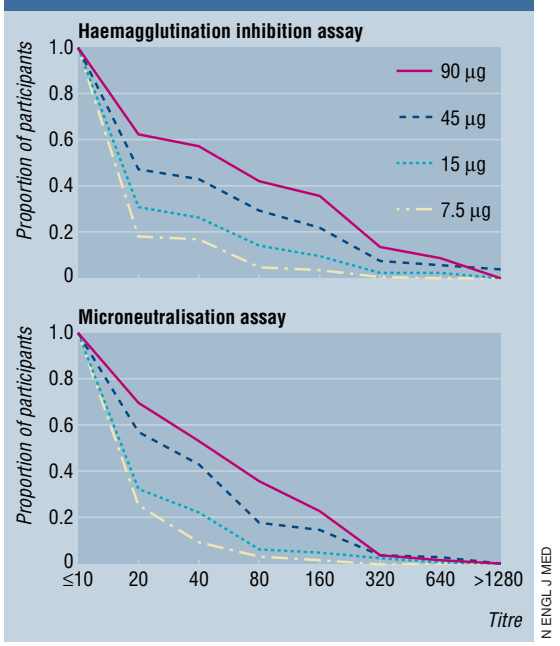

A safe effective vaccine is the best protection against a pandemic of avian influenza, and many vaccines are already under development. The most recent to be tested in humans was made in a similar way to standard influenza vaccines in the hope that the licensing would be relatively quick and easy if it worked. But it may not work well enough to be useful, a trial in healthy volunteers has shown. The vaccine was made from purified virions and was immunogenic only at the highest dose. Lower doses were significantly less immunogenic, and a dose equivalent to a standard influenza vaccine gave adequate antibody response in only $20 \%$ of volunteers.

Because the world's capacity for manufacturing these vaccines is limited, a higher dose per vaccine means that fewer vaccines can be produced. An editorial (pp 1411-3) estimates that we could produce only enough of this one to vaccinate 75 million people or $1.25 \%$ of the world's population. Meanwhile, the H5N1 virus is busy mutating into antigenically distinct strains, including a new one that recently killed a pregnant Chinese woman (pp 1421-2). A single vaccine is unlikely to be active against all of them.

N EnglJ Med 2006;354:1347-51

\section{Gatifloxacin is associated with serious disturbances in blood sugar}

The fluoroquinolone antibiotic gatifloxacin was launched in 1999. Since then, circumstantial evidence has been accumulating that it can seriously disturb glucose metabolism. Two large case-control studies settled the matter last week when they showed that gatifloxacin was associated with a fourfold increase in the risk of hospital treatment for hypoglycaemia (788 cases; adjusted odds ratio $4.3,95 \%$ CI 2.9 to 6.3 ) and a 16 -fold increase in the risk of hospital treatment for hyperglycaemia (470; $16.7,10.4$ to 26.8 ) in older people.

Both studies were nested in a large cohort of Canadians aged at least 66, who had taken macrolides, such as erythromycin; oral cephalosporins, such as cefuroxime axetil; or the fluoroquinolones gatifloxacin, levofloxacin, moxifloxacin, or ciprofloxacin. Using macrolides as a reference treatment, gatifloxacin was the only antibiotic strongly associated with metabolic disturbances. Levofloxacin was weakly associated with hypoglycaemia alone (1.5, 1.2 to 2.0$)$

The authors and a linked editorial (pp 1413-5) agree that the evidence against gatifloxacin should compel doctors to think again before prescribing it. For any given indication (commonly infections of the lower respiratory tract), equally effective, safer, and cheaper alternatives exist. In February this year, regulatory authorities warned doctors not to give gatifloxacin to anyone with diabetes.

N Engl J Med 2006;354:1352-61

\section{Simple infections may trigger venous thromboembolism}

A simple infection of the urinary tract or upper airways increases the risk of deep vein thrombosis and probably pulmonary embolus too. An analysis of routinely collected data from 220 UK general practices found a clear link between these two acute infections and venous thromboembolism that peaked in the first couple of weeks after infection and took up to a year resolve.

The researchers looked at the timing between infection and thromboembolism in 7278 patients with a first deep vein thrombosis and 3755 patients with a first pulmonary embolus. They found a twofold increase in the risk of either thromboembolic event in the two weeks after an acute urinary tract infection. The incidence ratio for deep vein thrombosis was 2.10 (95\% CI 1.56 to 2.82) and for pulmonary embolus was 2.11 (1.38 to 3.23). Respiratory infections, excluding sore throats and colds, were also associated with later deep vein thrombosis, with an incidence ratio of 1.91 (1.49 to 2.44) in the two weeks after infection. The authors think that these associations are probably causal and mediated by the effects of infection on blood coagulability, venous stasis, endothelial function, or all three. Acute infection should be added to the list of risk factors for venous thrombosis.

Lancet 2006;367:1075-9

\section{Bleeding is still leading cause of maternal death in Africa and Asia}

CAUSES OF MATERNAL DEATH, 1997-2002

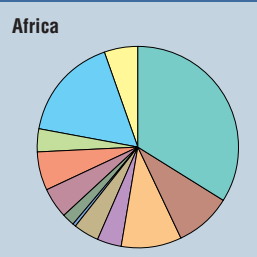

Asia

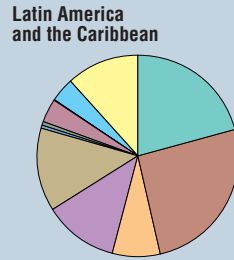

Developed countries
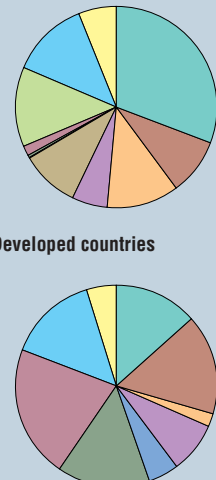

Unclassified deaths Other indirect causes of death Anaemia

Other direct ca

Embolism

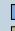

Ectopic pregnancy Obstructed labour $\square$ Abortion Sepsis/infections Haemorrhage

Pregnancy is still a serious hazard for many women in the developing world, and making it safer is a high priority for everyone. Current strategies for preventing maternal deaths are based on ageing data from more than a decade ago, however, so researchers from the World Health Organization did a systematic search for more up to date surveys, projects, studies, and statistics on how women die during and up to a year after pregnancy.

After filtering more than 4600 citations, they analysed 34 separate data sets from the rich and poor world. As expected, they found that bleeding was the leading cause of maternal deaths in Africa (34\%) and Asia $(31 \%)$, accounting for about a third of all deaths. Bleeding was also a common cause of death in Latin America and the Caribbean (21\% of deaths), but hypertensive disorders were the biggest killers here, responsible for $26 \%$ of deaths. Other notable geographical differences were the high proportion of HIV related deaths in Africa $(6 \%)$, the concentration of abortion related deaths in Latin America and the Caribbean (12\%), and deaths in Asia because of anaemia (13\%) or obstructed labour (9\%). Deaths due to infection and sepsis were common throughout the developing world.

\section{Lancet 2006;367:1066-74}

Alison Tonks associate editor atonks@bmj.com 